\title{
LA RIMA IX DE GUSTAVO ADOLFO BÉCQUER $\left(^{*}\right)$
}

\section{A Manucla.}

Entre las rimas de Bécquer algunas lan resultado especialmente atractivas a los investigadores, que han aplicado a ellas su atención y su esfuerzo. Es el caso, por ejemplo, de la Rima XV, y lo es el de la Rima IX. Ha sido analizada y estudiada, sin duda porque presenta carácter de plenitud y colerencia, de algo acabado dentro de la pequeñez de sus ocho versos. Ya se ha asegurado que "es ésta una Rima extraordinariamente interesante, además de ser una de las más bellas, por su simbólica humanización de lo cosmológico y por la cumplida adecuación de los elementos en unidad métrica, rítmica, de sonoridad, de imágenes y de contenido" ${ }^{1}$. Varios autores se han ocupado de ese contenido, de ese fondo que deja entrever la Rima, y han buscado también conexiones con otros textos que directa o indirectamente pudieran ser fuentes. Sin embargo, parece necesario resumir y poner de acuerdo los hallazgos de unos y de otros, para añadir y completar algunos aspectos que hayan podido quedar más olvidados.

\section{Texto.}

Junto al interés que of rece la Rima IX en si, hay un hecho que puede tener trascendencia desde el punto de vista de la actividad de Bécquer y su trayectoria poética. Iiste hecho fue planteado por Rafael de Balbín,

* Fste artículo turo su origen en un trabajo del Seminario de Critica literaria, dirigido por el prof. Dr. D. Rafael de Balbin, a quien he de agradecer mis escarceos iniciales por el mundo de la poesia de Bécquer. Posteriormente el trabajo ha ido creciendo con la incorporación de los estudios que sobre la Rima IX han veniclo publicando diversos investigadores, hasta el momento presente en que los refundo. Agradezco asimismo a mi buen amigo el Dr. J. M. a Díez Taboada la atención que ha dedicado al artículo, asi como las interesantes $y$ acertadas sugerencias que ha tenido a bien hacerme.

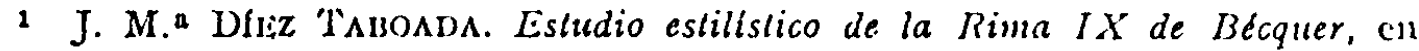
Bolclin de la Sociedad Castellonense de Cullura, 1958, abril-junio, XXXIV, p. IoG. 
al descubrir la versión de la Rima IX publicada en el Almanaque de El Museo Universal para 1868, con variantes respecto a la del Libro de los Gorriones ${ }^{1}$. Essta última versión dice así:

$$
\begin{aligned}
& \text { Besa cl aura que gime blandamente } \\
& \text { las leves ondas que jugando riza; } \\
& \text { el sol besa a la mube en Occident?. } \\
& y \text { de pirpura } y \text { oro la matiza; } \\
& \text { la llama en derredor del tronco ardiente } \\
& \text { por besar a otra llania se desliza. } \\
& \text { y husta el sauce inclinaindose a su peso. } \\
& \text { al rio que le besa, vuelve "un beso } 2
\end{aligned}
$$

La versión del Almanaque presenta notables variantes en los dos. primeros versos:

$$
\begin{aligned}
& \text { Besa el aura, que gime en son doliente, } \\
& \text { las crespas undas que en su vuelo riza: }
\end{aligned}
$$

Suponiendo, como lucgo ya comprobaremos, que la Rima IX es de composición temprana, podemos preguntarnos a qué se debe que Bécquer la publique tarde, y aún la corrija, estableciendo variantes, cuando al año siguiente la transcribe en el Libro de los Gorriones. ¿Qué tiene de particular esta Rima? ". Vamos a verlo.

\section{II.-Mundo poético.}

En primer lugar advertimos que en la intención de Bécquer este poema tenía una relevancia propia y particular. $R$. de Balbín, en un nuevo artículo, destaca el carácter unitario que presentan algunas de las Rimas que constituyen lo que él llama el cancionero menor de Bécquer, es decir, los quince pocmas que Gustavo llegó a publicar por sí mismo; y dice que "todos los poemas ven reforzada la unidad de su intención expresiva con la configuración fonológica y unitaria que la estrofa

- La publicación de las Rimas $I X$ y LIX de G. A. Bécquer, en Revisla de Lileralucra, 1955. VII, pp. Iy-29.

2 Libro de los gorriones, manuscrito 13.216 de la Biblioteca Nacional de Madrid. La Rima IX aparece en el lugar 27, fol. 553. Rubton BENítio hace notar que en esta versión el pronombre personal objeto masculino que Bécquer usa, verso $8 .^{\circ}$, es le y no lo. (Bibliografia razonada de $G$. $A$. B., IBuenos Aires. Universidad de Buenos Aires, I061).

3 Album poético, ell el Almanaque de I:l Museo Universal para 1868, p. 60.

- R. DE BAT.Bin. Arliculo cilado, p. 20. 
significa. I,a maestría configuradora de Bécquer goza agotando las fórmulas de unidad rítmica. Con la forma más densa y estricta de expresión unitaria quedan en el Libro dc los Gorriones dos poemas monoestróficos: la Rima XXIII y la Rima IX, dedicadas a uno de los tópicos favoritos del poeta y configurada la última sobre un paradigma estrófico, de tan cerrada estructura y culto abolengo como la octava real" ${ }^{1}$. Esste carácter unitario del ritmo parece quedar más realzado todavía si contemplamos al mismo tiempo el tema dentro del conjunto de las Rimas del cancionero menor. Díez Taboada hace el estudio desde este punto de vista temático, como Balbin lo había hecho desde el rítmico, y nos damos cuenta ahora del lugar importante que dentro de la concepción poética de Bécquer ocupa la Rima IX y su motivo del beso ${ }^{2}$. La Rima IX "expresa plenamente la cosmologización del beso, la concepción del beso como ley gencral del Universon. El beso logra en Bécquer una gran trascendencia. "Su idealismo y su pureza alcanzan unas dimensiones que no están en otros autores contemporáncos, $y$ menos que en ninguno en Heine, cuya concepción del beso no pasa de lo anécdótico y del mero juego de ingenio sensual" ${ }^{3}$. Lil mismo bécquer explica que "el amor es la suprema ley del Universo; ley misteriosa por la que todo se gobiena y rige, descle el átomo inanimado hasta la criatura racionaly". Todo el Universo en su plenitud muestra "un moviniento armónico, cuyo compás es el beso", el cual es también para Bécquer el eje ordenador de su "visión del Universo como totalidad que se realiza en el amorn. Un mundo animado que sirve no sólo a la expresión del anor del poeta, sino que siente en sí mismo los lazos de amor, de un amor "que inunda y nutre la naturale\%a entera" ${ }^{5}$. La presencia de ese amor hace que vibren todos los elementos naturales, tal como lo expresa Bécquer en la Rima X. Kin esta Rima se describe el fenómeno del amor universal de los elementos de la naturaleza, no como ley general - Rima IX-, sino cono tal proceso cósmico-amoroso, sorprendido en su realización progresiva, la

- Unidad ritmica y poema en el Cancionero menor de G. A. Bécquer, en Studia Philologica. Homenaje a Dámaso Alonso, t. I, Madrid, Gredos, 1960, p. I 42.

2 La mujer ideal. Aspeclos y fuentes de las Rimas de G. A. Bécquer, Madrid, C. S. I. C., 1965, pp. 102-106.

3 Dinz Tabonida, ibidem, pp. ro4-105. Un resumen sobre el motivo del beso en las Rimas puede verse en Dírz TABOADA, La Rima $X$ de G. A. Bécquer, en Bolelin Cultural de la Embajada Argentina, Madrid, marzo I963, num. 2, pp. I6- 7 .

- Cartas Literarias a nna mijer. III, cn $O$. C., I $3^{3}$. ed., Madrid, Aguilar, 1909, 1). 629 .

- J. R. Dínz, G. A. Bécquer. Vida y poesia, 2. ed., Madrid, Gredos, 1964 , pp. 408 y 398. 
cual se basa en una equiparación entre el paso del amor, que conmueve todos los elementos de la naturaleza, y el fenómeno del amanecer. La Rima X está en relación, por concepto y expresión, con otra serie de Rimas en las que se describe el amanecer y que aluden al beso en una función semejante a la que aparece en la Rima IX, y con expresiones parecidas'.

Iin la Rima $X$ se advierte una simbología sexual, referida al ciclo como elemento masculino y a la tierra como elemento femenino ${ }^{2}$. También en la Rima IX Díez 'Taboada ha advertido un simbolismo sexual, expresado, con mayor o menor precisión, en los cuatro pares de versos en los que aparecen los sustantivos: el aura/las ondas, el sol/la mube, la llama / otra llama (no el tronco, como recogia Esquer), el sauce / el río. Lil mismo autor añade que en cada uno de estos pares se muestra una edad de la vida, o sea respectivamente, la adolescencia, la juventud, la madurez, la vejez, con las características psíquicas propias del amor en cada una de esas edades. La indeterminación y levedad del adolescente, $c l$ aura, que ama jugando objetos femeninos plurales, las ondas; el ardor y luminosidad del joven, $c l$ sol, que colora y renueva la forma blanda e inconsistente de lo femenino, la mube; la identificación cle los dos elementos, masculino y femenino, en el amor de madurez, llama-llama, unidos ambos a la misma realidad vital, el tronco ardiente; la debilidad y languidez del viejo, el sauce, capaz sólo de devolver amor al rio juvenil y dinámico que le da el suyo. Naturalmente, la existencia de sustantivos neutros en español hubiese llevado el simbolismo sexual, en el caso de la madurez, a sus últimas consecuencias ${ }^{3}$.

\section{Estructura granatical.}

Lo anterior nos sitúa ya en el plano gramatical, que es el aspecto menos estudiado hasta ahora por los diversos críticos, y al que por ello vamos a dedicar aquí un particular análisis. Hasta ahora la crítica ha hecho una afirmación general sobre la waridez sintáctica de la enunciación" en nuestra Rima, que contrasta con "las formas dialogales" de las Rimas XX, XXIII y XXIX, que tienen también por motivo central el beso 4 . A este respecto viene muy bien recordar que la Rima $X$ co-

\footnotetext{
Dinz 'Tabonda, articulo citado sobre la Rima X, pp. Io-12.

Dfizz Taboaina, fbidem, pp. 77 y is.

Articulo citado sobre la Rima IX de Bécquer, p. 107.

- R. DI: BAL,Bfi, La publicrición..., p. 22.
} 
mienza a alejarse de la mera forma entuciativa y a introducir un conato de forma dialogada en aquella frase de su verso octavo, suprimida en la primera edición, pero presente en el Libro de los Gorriones:

$$
\text { - ¿Dime?... ¿Silenciol- ilis el amor que pasa! } 1
$$

Se ha aludido tanbién al léxico artificioso y rebuscado de la Rina IX, en lo que se refiere sobre todo al empleo de los sustantivos aura, asociado al verbo gemir, y sance. A tal fin, Balbin recoge y estudia los lugares paralelos que ofrecen estos motivos en otros textos del primer Bécquer, a los que luego nos referiremos ${ }^{2}$. Lil resultado es situar la Rima IX dentro del momento de su temprana composición, cuando Gustavo se ve envuelto en unas formas poéticas y un léxico de marcado carácter neoclásico, o imitador de él. Uni análisis, sin embargo, más atento descubre que el vocabulario de la Rima IX reúne sin duda palabras típicas del lenguaje neoclásico, pero junto a otras más generalmente literarias, de modo que en los sustantivos, frente a aura, occidente, purpura, onda, sauce, habría que poner sol, oro, llama, lronco, peso, rio y beso; y en los verbos, frente a matizar, deslizar, gemir e inclinarse a, más cultos, habría que considerar menos convencionalnente literarios besar, jugar, rizar, volver (devolver sería todavía más común).

La distribución del vocabulario en la Rima contribuye a la impresión de cerrazón y redondeamiento, pues comienza por el verbo besa y acaba por el sustantivo beso. En cuanto a los clemás pares de versos, se inician uno tras otro con los nombres de los distintos elementos de la Naturaleza.

La disposición de los verbos en la Rima es casi perfectamente simétrica y en ella apreciamos que el verbo besar va colocado casi siempre en la primera parte del verso, pero retardando cada vez más el acento, que recae en la $I^{a}$ sílaba del primer verso, en la $3 .^{a}$ sílaba del tercer verso, en la $3 .^{a}$ sílaba del sexto verso y en la $6 .{ }^{a}$ sílaba del octavo verso. Ein el sexto verso, aunque se acentúa en la tercera sílaba, el efecto que produce es el de haberse corrido el acento una sílaba, pues en el tercer verso se acentuaba bésa y en el sexto besir. En la segunda parte de los versos aparecen colocados los verbos en lugares correspondientes, gime (verso primero) y vulelve (verso octavo), jugando (verso segundo) e inclinándose (verso séptimo), y al final de los tres versos pares, los tres versos rimados, riza, matiza y desliza.

1 Dfiz 'l'

2 R. Dl: BAI, BfN, La publicaciún..., pp. 22-23. 
De modo que, vista en esquema, la distribución de los verbos en la Rima queda de la siguiente forma:

\section{SI L A B A S}

\begin{tabular}{|c|c|c|c|c|c|c|c|c|c|c|c|c|}
\hline & & 1.0 & $2 .^{3}$ & 3.2 & $44^{2}$ & $5^{2}$ & 6.A & $7 \cdot^{n}$ & $8 .{ }^{\circ}$ & $9 .{ }^{\circ}$ & 10.4 & $16, \mathrm{n}$ \\
\hline & 1.0 & Bé & sa & & & & gl & me & & & & \\
\hline & 2.0 & & & & & & & ju & gain & do & $\mathrm{ri}$ & $z: t$ \\
\hline v & $30^{\circ}$ & & & be & sa & & & & & & & \\
\hline $\mathbf{R}$ & 4.0 & & & & & & & & & ma & ti & z:t \\
\hline s & $5 .{ }^{\circ}$ & & & & & & & & & 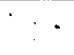 & $\because$ & \\
\hline s & 0.0 & & be & súr & & & & & & des & II & \%a \\
\hline & 7.0 & & & & $i_{n}$ & cli & nán & do- & & & & \\
\hline & $8.0 \mid$ & & & & & & bé & sa & vué & ve & & \\
\hline
\end{tabular}

Vemos, pues, en el esquema que el verbo maliza ocupa el centro de la composición. Antes de él, el poema presenta dos veces el verbo besar y después de él, otras dos. $\Lambda$ simismo los gerundios se distribuyen, uno en la parte superior y otro en la inferior. Igualmente el verbo gime se corresponde con vuclve y los consonantes riza/desliza se ligan por la rima impuesta por el esquema estrófico.

Eil tiempo de los verbos es siempre un presente permanente, que en realidad señala más bien un aspecto de intemporalidad, ya que se trata de describir un comportamiento de la Naturaleza. El carácter descriptivo exige precisamente el modo indicativo en que están todos los verbos, modificado únicamente por las tres formas de gerundio e infinitivo. Esta matización modal se logra en el primer verso con un adverbio de modo, el único de toda la Rima, blandamente, y en cuanto a otras referencias se reducen a meras indicaciones locales, en Occidente, en derrclor del tronco, o materiales, de prirpura $y$ oro ${ }^{1}$.

Respecto a la sintaxis oracional, cada frase está incluida en un par de versos sin encabalgamiento alguno y liay que señalar la mayor complicación sintáctica en el primero y en el último. En el primero, además de la oración principal, hay dos adjetivas explicativas de relativo, aparte del gerundio. En el último par, además también del gerundio, aparece igualmente una oración adjetiva de relativo, referida al complemento directo, $y$ con distinto sujeto al de la oración principal. Los dos

1 Dfez 'TABond, articulo citado solse la Rina IX, pp. 107-108. 
pares centrales, segundo y tercero, presentan asimismo una gradación que va de la coordinación copulativa del segundo a la subordinación (por besar) del tercero; pero enlazan respectivanente el segundo con el primero en cuanto a los dos verbos de cada uno (besa y riza, besa y matiza), y el tercero con el cuarto en que desaparece la claridad de besa como oración principal, sustituida en el tercero por besar, y en el cuarto atribuida al complemento directo. En estos dos pares últimos se da más claramente el hipérbaton que en los dos primeros, de modo que también en este punto se produce una progresión. Firente a la trasposición del sujeto en el primer verso (besa el aura) y la anteposición del complemento circunstancial en el segundo miembro de la coordinada ( $y$ de pirpura y oro la matiza), está el hipérbaton del tercer par de versos, en el que la primera palabra (la llama) es el sujeto, y la última (desliza) es el verbo, y el del cuarto par de versos, con el complemento antepuesto y convertido en indirecto (al rio que le besa, vuelve un beso).

Para mejor ver los efectos de la trasposición y del hipérbaton, basta tener en cuenta el orden lógico de las frases, que más o menos sería así:

I:l autra, que gime blandamenle, besa las leves omlas, que riza jugando, el sol besa a la mube en Occidente y la matiza de pirpura y oro, la llama se desliza en derredor del tronco ardiente por besar a otra llama $y$ hasla el sauce, inclindindose a su peso, vuclve un beso al río que le besa.

Aún se da en la Rima otro efecto, debido también al juego de la trasposición y del hipérbaton, y es la impresión de dinamismo que la simple lectura del poema produce. Esta sensación dinámica pensamos que se podria atribuir también a la abundancia de verbos. Sin embargo, al hacer el recuento, nos encontramos con que hay catorce sustantivos frente a once verbos solamente ( 24 por Ioo frente a I9 por I00). De todos modos, si atendemos como antes a la colocación de los verbos, veremos que están casi siempre destacados de algún modo; por acentos fundamentales, o por acentos antirrítmicos, o por acentos extrarrítmicos, en primera sílaba de verso, como veremos más adelante.

Los verbos de los cuatro primeros versos de la Rina contrastan con las formas reflexivas de los tres últimos versos (se desliza, inclinándose, que le besa, ésta sólo psicológicamente reflexiva, ya que el le complemento se refiere al sujeto de la oración principal). Estas formas reflexivas están precisamente en los versos que expresan las edades de madurez y vejez, de acuerdo con el simbolismo que hemos indicado anteriormente. E1 pronombre le es una muestra del carácter leísta normal en la sintaxis de Bécquer. Por otra parte, la personalización tan acu- 
sada en esta Rima de los elementos de la Naturaleza, se muestra claramente en el uso de la preposición a ante complementos directos de cosa, cxcepto el caso de ondas, que representa el amor de la adolescencia, donde un encuentro seriamente personal no se realiza todavía.

Las correcciones de la versión del Libro de los Gorriones, que sustituyen a las expresiones del Almanaque del Museo Universal, añaden mayor variedad sintáctica a la Rima al convertir los sintagmas en son dolicnle del primer verso y en su vnelo del segundo verso en blandamente y jugando respectivamente, al mismo tiempo que cambian crespas por leves. De este modo se afianza el sentido de toda la frase del primer par de versos, y a la vez se evita la excesiva repetición de construcciones demasiado semejantes, ya que las dos expresiones sustituidas, en son doliente y en su vitelo, se parecían demasiado a en derredor del tronco ardicnte del verso quinto ${ }^{1}$.

Iin cuanto a los aspectos semánticos, lo más importante es señalar los diferentes matices del significado del verbo besar en cada uno de los cuatro pares de versos: en el primer par besar significa un levísimo 'roce' superficial, como el del vicnto suave, aura, que riza las ondas del mar; en el segundo par besar adquiere, en cambio, un sentido de envolvimiento' (recuérdese la expresión paralcla de la Rima IV, versos 7-8: "micutras el sol las desgarradas mubes / de fuego y oro visla"), por medio de matices y colores (puipura $y$ oro) con que $c l$ sol cubre las formas poco definidas aún de la nube cn el ambiente del crepúsculo ${ }^{2}$; en el tercer par, besar se carga del sentido de 'fusión' íntima, más aún, de identificación absoluta de dos elementos que pertenecen ya a una misma realidad (el tronco ardiente); en el último par se da una reiteración, besar / beso, (aumentada por la aliteración de la rima peso), que indica el carácter de insistencia en el encuentro del elemento pesado o sólido con el fluido, buscando una fusión que sólo llega a ser 'impregnación'. Se ve con claridad la gradación semántica intensiva en la matización del significado del verbo besar, la cual, por otra parte, está totalmente de acuerdo con el sentido y estructura temática que descubrió Díez 'l'aboada en la Rima IX: cuatro edades de la vida del hombre, personificación de la Naturaleza.

1 Sobre los dos prineros versos de la Rima IX en sus dos versiones, véase el articulo de Díz TABonda en Homenajes. Estudios de Filologia Española, III, que aparecerá próximamente.

- İin relación con el crepisculo en Bécquer, véase lo que acerca de la Rima XXXII dice Díz TABOADa en La mijer ideal, pp. 23-24. 
IV. Estructura ritmica.

lîstá claro que Bécquer ensayó diversos metros clásicos tradiciouales, casi todos en una sola estrofa, haciendo un verdadero muestrario, muy propio del carácter de producto de laboratorio que las Rimas presentan. Así la seguidilla (Rima LXXVIII), la quintilla (Rima LX), el serventesio (Rima XX), etc. Aquí, cu la Rima IX, la octava real ${ }^{1}$. A estos poemas monoestróficos clásicos habría que añadir la Rima XXIII, que recoge una copla popular asonantada. Ya nos hemos referido a la forma "densa y estricta de expresión unitaria" y al "abolengo culto" de la estrofa de la Rima IX ${ }^{2}$. La octava real, a pesar de su clasicismo, fue muy bien acogida por los primeros románticos, sobre todo por aquellos que poscían una más sólida formación clasicista. La solian usar para temas que ellos concebían cono clásicos, como la descripción de la Naturaleza o de la mujer. In cuanto a Bécquer, sólo tiene esta octava real en las Rimas, aunque es verdad que escribió otra octava, bermudina, que constituye la Rima $A$ Casla, y que otra vez hizo una octava en la Oda a Quintana, La Corona de Oro (versos 234-242), alterando el verso final:

$$
\begin{aligned}
& \text {... es el asombro de la musa hispana? } \\
& Y \text { el eco murmurando } \\
& \text { me respondio fugaz: "lise es Quintana". }
\end{aligned}
$$

Con variaciones importantes, tres rimas consonantes en vez de dos en lus seis primeros versos, Bécquer ronda también la octava real, perosin ajustarse tampoco al canon tradicional en el comienzo de la Rima LVII. En esta Rima Gustavo usa un esquema $A B C A B C D D$, en lugar de $A B$ $\mathrm{AB} \mathrm{AB} \mathrm{CC}^{3}$.

La estructura ritmica de la Rima IX se corresponde perfectamente con la sintaxis y el contenido. Hay cinco acentos extrarrítmicos en tercera y uno en primera silaba. De esos cinco extrarritmicos en tercera, uno es antirrítmico. 'Tanto el extrarrítmico en primera (primer verso) 4 , como el antirrítmico (tercer verso) y otro de los extrarrítmicos en tercera (verso sexto), destacan formas del verbo besar. Los otros extrarrítmicos

1 Véase G. A. Bricquirk. Rimas. Listudio y edición de J. M. a Dirz TanondA, Maulricl, Alcalii, 1965,1 1. 32.

2 R. DI: BAI,BfN, Unidad ritmica y poema..., p. I42. Véase nota $x$, p. 229.

3 R. DIE BAIBfN, La publicación..., pp. 20-2I, Díbz TABOADA por su parte demostró que esta Rima I,VII se relaciona precisamente con una octava real del Canto III de El diablo mundo de lispronceda. Véase La mujer ideal, p. I 35.

- Dinz Tauonda, en su articulo citado sobre la Rima IX (p. 1ว7) llama a este acento antirritmico, siendo en realidad extrarritmico. 
en tercera sílaba (verso primero, verso cuarto y verso séptimo), ponen de relieve palabras clave como aura, pirpura, sauce. Estas tres palabras ofrecen la coincidencia de referirse a elementos indecisos, crepusculares ${ }^{1}$. Las palabras que indican elementos luminosos plenos, sol, llama, tienen acentuación rítmica. Ya anotábamos antes el retardamiento en la acentuación que sufría el verbo besar en el primero, tercero, sexto y octavo verso. En este último la forma besa se abrocha con el sustantivo beso, muy de acuerdo con el esquema métrico cerrado de la octava real.

Ein la segunda parte de la Rima los últimos cuatro versos of recen un esquema ritmico perfectamente simétrico: con acentuación en segunda, sexta, octava y décima sílabas los versos quinto y octavo; y en tercera, sexta y décima los versos sexto y séptimo.

Respecto a la sonoridad de la Rima es enormemente acusada. Díez Taboada anotó ya la serie detallada de los juegos de aliteración y su sentido ${ }^{2}$.

V. Futentes.

Ramón Esquer recuerda unos versos de la tigloga I de Garcilaso a propúsito de la Rima IX en sus versos tercero y cuarto:

\author{
Nunca pusieran fin al lrisle lloro \\ los pastores, $n i$ fueran acabadas \\ las canciones que sólo el monle ola, \\ si mirando las mibes coloradas. \\ al tramontar del sol bordadas de oro. \\ no vieran que era y'a pasado el dia ${ }^{3}$
}

Pero mucho más importante que este leve contacto es la influencia de Espronceda que el mismo Esquer analiza. En concreto relaciona nuestra Rima con la octava quinta del Canto IV de El Diablo Mundo, $\mathrm{y}$ hace un detenido análisis destacando la semejanza no sólo de metro, sino también de contenido:

Las rosas sobre el tallo se levantan

coronadas de gotas de rocio,

las avecillas revolando cantan

al blando son del murmuturar del rio;

chispas de licz los aires abrillantan,

salpicando de oro el bosque 3 mbrio;

y si el aura a la flor murmura amores.

la flor le brinda aromas $y$ colores.

1 Véase la cita de la nota 2 de la p. 234.

2 Artículo citado sobre la Rima IX, pp. ro8-109.

3 BRAE, 1965, XI,V, pp. 188-189 


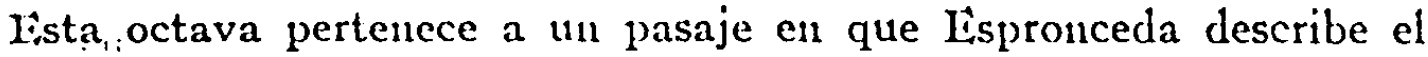
amanecer, imitando el lenguaje neoclásico, para acabar burlándose de lo mismo que ha escrito '. Díez Taboada habia relacionado antes este mismo pasaje con la Rima XV, que guarda con él estrecho contacto, y también con la Rima X, por el parecido del verso quinto de esta octava, "chispa de luz los aires abrillantan", con una de las variantes de la Rima X; todo esto nos hace suponer un punto de partida y aún un fondo común para estas tres Rimas, IX, X y XV, relacionadas con el proceso cúsmicoamoroso de que al principio hablábamos, comparado precisamente con el amanecer, que es, como decimos, el fenómeno que se describe en la octava de Espronceda ${ }^{2}$.

Lil influjo de la octava de Lispronceda sobre la Rima IX es evidente, aunque no se pueda en este caso apurar tanto al análisis como Esquer pretende. Las coincidencias entre los dos pasajes que Escquer llega a fijar son:

x. ${ }^{\circ}$, ritmo yámbico en casi todos los endecasílabos y abundancia de acentos antirrítmicos.

2. ${ }^{\circ}$, estructura bipolar en la octava de Espronceda semejante a la que Diez 'Taboada scĩala en la IX, e incluso una progresión cronológicoamorosa que describe el despertar. Iisquer fucrza un tanto las cosas para llegar a ver esta progresión. Precisamente lo que Bécquer añade a lispronceda es un rigor mucho más estricto, como ya lo liabía señalado Bunsoño ${ }^{3}$.

$3 .^{\circ}$, sustancial coincidencia del vocabulario: de veintinueve palabras portadoras de sentido en la octava de Espronceda y veintiocho en la Rima de Bécquer, coinciden cuatro en la versión del Libro de los Gorriones; aura, oro, rio, blando / blandamente, y cinco en la primera versión del Almanaque de El Museo Universal, tres de las palabras ya citadas, menos blando; y además son y vilelo / revolando. Otras semejanzas de vocabulario que Esquer señala no son tales. Sí lo son, en cambio, las que presenta la Rima IX con la tercera y cuarta octava del mismo pasaje inicial del Canto IV:

Indcese el sol...

$y$ de naranja y oro $y$ fuego pinta...

1 Versos $2,177-2,184$ y taubién las estrofas anteriorcs $y$ siguientes. Véase K. IisQuir, Presencia de Espronceda en Becquer, en $R I E E$, I96.3. XIVI, pp. 329-341.

2 Véase Dfuz 'TAnonds, La mujer ideal, p. $30 \mathrm{y}$ el articulo citado sobre la rima $\mathrm{X}$, notas 7 y 20 .

- Los conjuntos paralelisticos de Bécquer, en Seis calas en la expresión literuria española. 4." ed. Madricl, Gredos, 1970, p. 201. 
en relación con los versos tercero y cuarto de nuestra Rima:

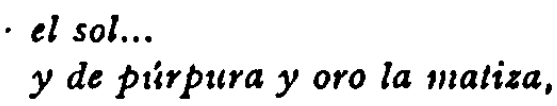

o con el verso paralelo de la Rima IV (v. 8):

de fuego y oro visla.

Lo mismo ocurre con

se desliza...

que jugando salla

en contacto con se desliza del verso sexto, y que jugando riza del $2 .^{\circ}$ de nuestra Rima ${ }^{1}$.

Lo que Esquer no añade es que Dlez Taboada había señalado ya cómo los consonantes de los versos pares de la Rima IX, riza, matiza, desliza, parecen estar seleccionados previamente, como hizo Bécquer con otras Rimas (la XV y la LXXIII) y cómo los tomó precisamente de otra octava de Espronceda, la comprendida entre los versos 3.94 I y 3.948 de El Diablo Minndo 2.

José Pedro Díaz relacionó la Rima IX con un pasaje de Rolla de Alfred de MIusset:

J'aime, lui dit la fleur, et je meure cmbrasé

des baiscrs du atphir, qui me relevera.

J'aime! - voild le mot que la nature entière

crie als vent qui l'emporte, à l'oiseau qui le suitl

Ramón Esquer rechaza totalmente este pasaje como fuente de 1a Rima IX ${ }^{3}$. Más probabilidades merece el contacto con Musset señalado por R. Pageard:
Mes chers amis, quand je mourrai, planlez un saule ans cimelière. J'aime son fenillage éploré, la pâleutr m'en est douce et chìre.
Et son ombre sera légère
a la terre oil je dormirai 4.

1 R. IisQuer, art. cit., pp. 337 y 338 . Remitimos al artlculo citado en la nota I, p. 234 .

La mujer ideal, p. 160, nota 13.

- J. l. UfAz, op. cil., pp. 283 y 284 . R. EsQUiE, art. cil., p. 334.

- Las Rimas de G. A. Bécquer. Edición cronoldgica y crilica (en preusa), fol. 312. Se trata del poema Lucie, mayo de 1835 . 
Ahora bien, esta fuente tampoco muestra un parecido suficiente con la Rima IX y sólo tiene alguna validez a la luz de otros textos de Bécquer con motivos semejantes a los de nuestra Rima, que señalamos inmediatamente.

\section{Contactos con otros textos becquerianos y génesis de la Rima.}

I a referencia a la muerte que establecen los versos más arriba citados de Musset, hace pensar en el pasaje de las Cartas desde mi celda en que Bécquer une también los dos motivos, saucc y tumba, haciendo notar, como linitación de la relación que establece Pageard, que Musset no menciona para nada el río en relación con el sauce, que es precisamente lo que constituye el motivo de los últimos versos de la Rima IX. El pasaje de la Carta III desde mi celda es el que citaba ya Esquer como lugar paralelo de la Rima. Dice así:

-...el sauce, cubriendo aquel lugar de una flotante sombra, le prestaria su vaga tristeza, inclinándose y derramando en derredor sus ramas desmayadas y flexibles, como para proteger y acariciar mis despojos, y hasta el ro, que en las horas de creciente casi vendria a besar el borde de la losi, cercada de juncos, parecería arrullar mi sueño con una música agrarlable».

Ein la misma Carta III Balbín cita también otro pasaje con el mismo motivo; responde al recuerdo que Bécquer dedica a sus paseos por la orilla del Guadalquivir y al deseo de que allí precisamente estuviese su tumba: "Un sauce baña sus raíces en la corriente del rio, hacia el que se inclina como agobiado de un peso invisible...." ${ }^{1}$.

Listas relaciones con la Carta III desde mi celda indujeron a Balbín a pensar que la Rima IX pertenece a la primera etapa de las tres que el mismo Bécquer describe en dicha Carta $I I I$, es decir, al período fundado en "motivos renacentistas y naturales", época temprana y juvenil. $Y$ esto por diversas razones: por el clasicismo de la forma, por el léxico próximo a los poemas anteriores a las Rimas y extraño al resto de su obra, por la alusión a la época sevillana de su vida, a la que pertenece también cl escenario aludido del sauce y el rio ${ }^{2}$. Esquer acepta estas razones de Balbín y añade algunas nuevas, teniendo en cuenta la influencia de la traducción que E. I. Sanz hizo del alemán, titulada $T u$, él y y'o, y que Balbín ha relacionado lógicamente con la Rima XV ${ }^{3}$. Ramón Essquer

1 Vease la nota 2, p. 23 de nuestro trabajo. Lil pasaje puede verse en $(; . A$. Bliceulik, $O$. C., ed. cit., Madrid, Aguilar, 1969, 1). 532.

2 La publicación......, pp. 20-25.

3 R. LSQULiR, art. cil., p. 338. R. DE BAI,BfN, Un influjo germanisla en Bécquer, en el ITomenaje a Johames Vincke, Madrid, 1962-63, pp. 85I-866. 
ve en este poema de Sanz cierto lenguaje no lejano de la Rima IX y de sus fuentes ya señaladas (utronco... cabe la fuente, sauce sombrio/que hacia la tierra dobla su frenten), y teniendo en cuenta todas estas cosás, llega a creer adivinar la génesis de la Rima, y explicar así la paradoja, que a Balbín ya le extrañó y que recordábamos al principio de este artículo, entre el hecho de la, al parecer, evidente composición temprana de la Rima IX, su tardía publicación ( 1867 ) y su más tardía corrección y reforma; al incluirse en el Libro de los Gorriones el año siguiente (18681869). Balbín la explicó por "el hondo y persistente arraigo, en la personalidad poética de $G$. $A$. Bécquer, de la extensa y demorada formación neoclásica, recibida en sus años de aprendizaje sevillano" ${ }^{1}$. Para R. Es(quer, lo mismo que para J. P. Díaz, J. M. Díez Taboada y R. Pageard ${ }^{2}$ tampoco of rece duda que la composición de la Rima IX sea temprana y juvenil, y a estas razones que da Rafael de Balbín sobre el recuerdo sentimental de sus años sevillanos neoclásicos, Esquer añade el influjo del pasaje también neoclásico del Canto IV de El Diablo Minndo de Espronceda y el de la traducción de E. F. Sanz, los cuales, por influir támbién en la Rima XV, hacen pensar a Esquer que el recuerdo, publicación y remozamiento de la Rima IX están en íntima conexión con la composición, recuerdo y retoques de la Rima $X V$, tan querida para Bécquer $y$ clave central de su mundo poético ${ }^{3}$. Esstas razones de Esquer aún pueden confirmarse si tenemos en cuenta las coincidencias del vocabulario de nucstra Rima con el de los poemas de adolescencia, anteriores a I855, y las coincidencias de motivos con la Oda a Quintana, publicada en IS55, y con la Historia de los tcmplos de España, que apareció en 1857 . I,os pasajes de la citada Oda a Quintana, que presentan contacto con la Rima IX, sorı:

y entre los sances de las tumbas gimen con dulce soplo las ligeras auras (versos 19-20).

Yo de la oscura elernidad dormia el dulce sueño, la cansada frente reclinando en un sance que crecla solitario en la orilla del torrente (versas 126-129) 4.

I La publicación..., p. 25.

3 R. Lisqur:R, art. cit., pp. 339-340. J. P. DfAz, op. cit., p. 340. J. M.\& Df́Ez IABOADA, articulo citado sobre la Rima X, nota I5, R. PAGEARD, op. cit. (en prensa), folio 307 .

- Díz 'Mdonda, Subre la Rima $X V$, en Revisla de Literalutra, I962, XXII, 1). If y nota 9 y también La mujer ideal, p. 30 .

- R. DE BALBfN, La publicación....., pp. 22-23. Coincide la Rima IX con los poemas de adolescencia titulados, Las dos, Elvira, Al cefiro. 
El pasaje de Los lemplos de España, al que nos referimos, pertenece al capitulo titulado Basilica de Santa Leocadia: "A nuestra izquierda, y escondiéndose por intervalos entre el follaje de sus orillas, el río se alejaba besanclo los sauces que sombrean su ribera...n" ${ }^{1}$.

Estas semejanzas de la Rima IX con otros textos becquerianos, indudablemente de primera época, corroboran el carácter temprano, dentro de la obra cle Gustavo Adolfo, que se atribuye a la composición de la Rina estudiada.

Iin cuanto a los contactos con otras Rimas, basta recordar la semejanza entre la expresión Besa el aura de nuestra Rima y beso del aura de la Rima $\mathrm{X} r$ :

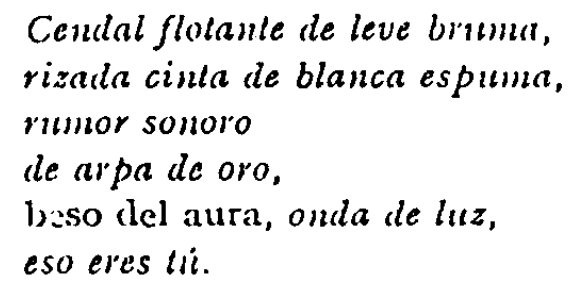

También aparece en la Rima XV la llama (v. 9), pero sin especial parecido con la expresión de la Rima IX. Eal verdadero parecido está en los cuatro primeros versos de la Rima Xxiv:

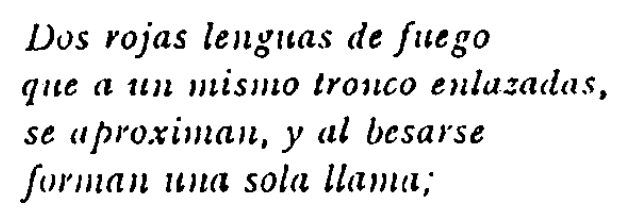

que desarrollan el mismo motivo, con expresión más concisa, de la Rima IX (versos 5 y 0 ) y explican claramente el valor semántico de "fusión", que atribuímos a esta imagen. La Rima LXVII en su tercera estrofa recuerda este motivo de las llamas, como lcnguas de fuego, pero sin que se aluda a su fusion por el beso. La referencia al beso está, en cambio, en la primera estrofa de esta misma Rima:

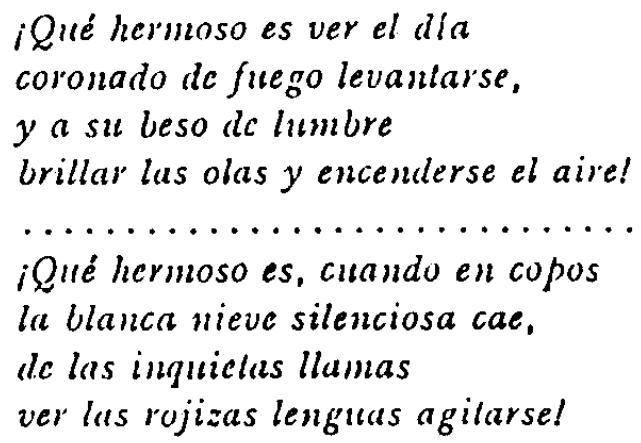

1 R. PAcionkd, op. cil., (cn prensa), folios 3ri-3iz. Lil pasaje recordado

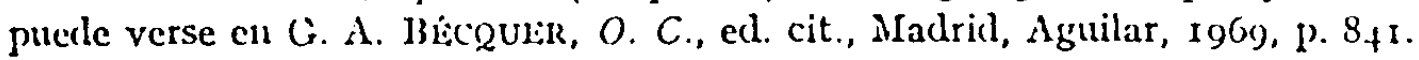


Los versos de la estrofa primera explican el proceso cósmico-amoroso a que nos referiamos al comienzo de este artículo, y que Bécquer desarrolla a lo largo de varias Rimas, tal y como Díez 'Taboada lo demostró ya, cuya base sensorial está constituida precisamente por el fenómeno del amanecer y uno de cuyos momentos, aún antes del amanecer, es el anhelo de fusión (Rima VIII):

...y con ellas (las estrellas)

en lumbre encendido

fundirme en un beso.

Dentro de este mismo proceso cósmico-amoroso queda incluido el sentido de los dos primeros versos de la segunda estrofa de la Rima IV:

Mientras las ondas de la luz al beso palpilen encendidas,

pero los dos segundos,

mientras el sol las desgarradas nubes

de fuego $y$ oro vista,

están ya en total contacto con el segundo par de versos de la Rima IX.

Por último, en la segunda parte de la Rima IV, hay un par de versos:

micntras sentirse puedan en "1n beso

dos almas confundidas,

cuyo contenido nos remite de nuevo a la Rima XXIV, cuya última estrofa expresa el mismo motivo de modo muy semejante:

dos besos que a un tiempo estallan... eso son muestras dos almas.

Essta concepción del amanecer como expresión del amor, que por lo demás se da también en la III de las Cartas literarias a una mujer, que antes citamos, responde al tono alegre y esperanzado que otros investigadores han descubierto en la Rima IX ${ }^{1}$, asi como en todos los poemas publicados personalmente por Gustavo.

Se ha relacionado esta concepción cósmico-amorosa de Bécquer con

1 R. DE BAL,BfN, La publicacionn..., p. 29. J. MI. ${ }^{2}$ DfEz TABOADA, La mujer ideal, p. 1oú. 
diversas teorias filosóficas. Es verdad que Bécquer estaba influido por un platonismo lírico tradicional español, que enlaza con el "circulo amoroson de León Hebreo ${ }^{1} \mathrm{y}$ también por todo un sentido romántico de aninización de la Naturaleza. Sin embargo, otras referencias de mayor alcance ligadas directamente a determinados sistemas filosóficos, habría que dejarlas, creemos, aparte. Bécquer no pretendía hacer filosofía, ni su formación le ayudaba a ello. Sus hallazgos y aciertos prodigiosos son de carácter poético y proceden de su portentosa intuición y fantasía para descubrir el lado esencial y bello del mundo?

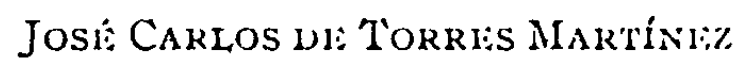

1 J. P. Dfaz, op. cit., pp. 387 y 396.

- R. DE Bar,BfN: El tema de España en la obra de Becquer. Oviedo, Universidad Literaria, 1944, pp. I-40. 